\title{
Psychotherapy for gender identity disorders ${ }^{\dagger}$
}

\section{Az Hakeem}

\begin{abstract}
SUMMARY
This article describes a special adaptation of group psychotherapy as a psychological treatment for people with a variety of gender identity disorders. It can be used as an alternative to or concurrently with hormonal and/or surgical interventions for transgender people. It is also suitable for individuals whose gender identity disorder remains after physical interventions. The article draws from a UK specialist pilot for such a treatment service and describes the explicit aims of the psychotherapy, the specialist adaptation of therapeutic technique required and observed thematic features relevant to working in this specific field.
\end{abstract}

\section{DECLARATION OF INTEREST}

A.H. runs the gender identity disorder service described in this article.

Although many clinicians are familiar with transgender individuals and the physical treatments available to them, many will be unfamiliar with the psychological treatment options available for other gender identity disorders. Eden and colleagues have detailed the role of the gender specialist when offering assessment and physical treatments in the forms of hormone therapy and surgery to transgender patients (this issue: Eden 2012; Wylie 2012). In this article I outline the specialist adaptation of psychotherapy as a treatment for people with gender identity disorders that do not neatly fit into a transsexual diagnosis or for whom physical sex (gender) reassignment is not a suitable option. The recommendations here are based on findings from a specialist psychotherapy service for patients with gender identity disorders that operates in the UK within the National Health Service (NHS).

\section{Setting up a specialist psychotherapy service for gender identity disorders}

Ten years ago a specialist psychotherapy service was set up in the UK for NHS patients with gender identity disorders. The service was initially run as a pilot with the aim of determining whether psychotherapy could be helpful to patients referred with gender identity disorders with regard to the problems they had sought help for from the clinic. The unique service, which operates from a small specialist psychotherapy clinic in London, is now available to patients from anywhere in the UK.

The clinic specialises in offering psychotherapy to people with problems relating to their personality, sexuality, gender, violence or delinquency. The clinic receives national specialist funding and patients may be referred by any health professional from primary, secondary or tertiary care services anywhere in the UK. As the clinic is based in London, most of the patients are referred from within the greater London area, which is covered by a block contract. A smaller number of patients are referred from the rest of the UK, many travelling several hundred miles each week for appointments. The health authorities for these individuals will have had to agree funding for assessment and treatment for these out-ofcontract referrals. The clinic is unique within the NHS in being able to offer patients time-unlimited psychotherapy, which is a recognition of the longstanding nature of many of the problems for which patients are referred.

I currently run the gender identity component of the clinic, allocating 1 day a week to the service. During this time I am able to carry out one patient assessment and run two therapy groups, each for eight patients. Thus, the capacity of the service is at present limited to 17 patients a week.

\section{Referrals to the service}

The usual presenting symptom of the individuals referred to the service for psychotherapy is a feeling of unhappiness or confusion with their sense of gender identity that they hope a talking therapy will resolve. If the gender identity disturbance is part of a transgender identity, the patients may also be considering physical treatments for sex reassignment, although this is not always the case. Some will have already had sex reassignment surgery but remain unhappy regarding their sense of gender.

Before the service was set up, it was unclear whether people with gender identity disorders would be interested in psychotherapy, would
Az Hakeem trained in both forensic psychiatry and psychotherapy before taking up his current consultant posts with Camden and Islington NHS Foundation Trust and the Tavistock and Portman NHS Foundation Trust. He is an acute in-patient consultant, the head of a mentalisation-based therapy service for borderline personality disorder and, since 2001, has run the UK specialist service offering psychotherapy for people with gender identity disorders. He has trained as a group analyst and has worked as a psychiatrist for the media on a number of television programmes. Correspondence Dr Az Hakeem, Highgate Mental Health Centre, Dartmouth Park Hill, London N19 5NX,UK.Email: az@drazhakeem. com

†See also pp. 2-11 and 12-16, this issue. 
persist with it and find it useful, and also what specialist adaptations to the therapeutic delivery would be required. However, we have found that the patients offered psychotherapy consistently stay with the intervention. They report it to have improved the stability of, and their satisfaction with, their sense of gender identity, whether this be their primary biological one, a transgender identity, or indeed a non-binary gender identity (when the individual chooses not to identify as male, female or transgender). The steps needed to corroborate these anecdotal patient-reported benefits in an empirical manner will be discussed later in this article.

\section{Literature review}

There is scarce literature available on the provision of psychotherapy for individuals presenting with gender identity disorders. A National Library for Health literature search limited to papers on 'transsexual', 'transgender' and 'psychotherapy' of any modality yielded just 29 papers, most published over three decades ago.

A major limitation of the early literature, which was psychoanalytically based, is that the theoretical models were extrapolated from small numbers of case studies, and then applied to larger populations that may have varied from the individuals in the original studies (Greenson 1968; Stoller 1968: pp. 89-125; Stoller 1975: pp. 39108). The specialist service that I run has to date assessed and/or treated about 100 patients, some having only a few consultations, others in longterm psychotherapy over many years. Although the patients referred share a presenting symptom related to their sense of gender identity, detailed assessment reveals considerable diagnostic heterogeneity (Table 1).

\section{TABLE 1 Diagnoses of 82 patients referred to the UK} gender identity psychotherapy service

\begin{tabular}{|c|c|c|}
\hline & Proportion & $n$ \\
\hline \multicolumn{3}{|l|}{ ICD-10 categorya } \\
\hline F64.0 Transsexual (pre-operative) & $61 \%$ & 50 \\
\hline $\begin{array}{l}\text { F64.0 Transsexual (post-operative) } \\
\text { with dissatisfaction/gender dysphoria } \\
\text { after sex reassignment surgery }\end{array}$ & $26 \%$ & 21 \\
\hline F64.1 Dual-role transvestism & $13 \%$ & 11 \\
\hline F65.1 Fetishistic transvestism & $15 \%$ & 12 \\
\hline Autogynaephilia ${ }^{b}$ & $5 \%$ & 4 \\
\hline F44.81 Multiple personality disorder & $2 \%$ & 2 \\
\hline Proportion of female to male & $17 \%$ & 14 \\
\hline \multicolumn{3}{|c|}{$\begin{array}{l}\text { a. Patients may code for more than one category. } \\
\text { b. Autogynaephilia does not appear in ICD-10 (World Health Organization } \\
\text { 1992). The condition refers to males who are sexually aroused by the } \\
\text { idea of themselves as women or having female attributes/bodily parts } \\
\text { (Blanchard 2005). }\end{array}$} \\
\hline
\end{tabular}

The literature on group psychotherapy for gender identity disorders yields only nine papers: Lothstein and his colleagues at the Case Western Reserve University Gender Identity Program in Ohio have described their work with expressive group therapy (Lothstein 1977a,b, 1979, 1981; Althof 1980) and in more recent years I have published in this area (Hakeem 2007, 2008, 2010a,b).

\section{The shifting focus of treatment}

Immediately apparent in the existing literature is the evaluative shift in focus over time. Earlier studies involved mostly born-males and were aimed at reducing 'effeminacy' or homosexuality, which were deemed undesirable features (Green 1973; Limentani 1989: pp. 133-154). Authors seemed to be confined to the limitations of a rigid repertoire of gender identities. This was in keeping with the narrow, binary experiences still prevalent in many transgendered patients, who feel that they have to be confined to one gender construct or switch to another. More recently, writers have conveyed a therapeutic attitude that is more accepting of uncertainty and ambiguity, rather than rigid adherence to both heterosexual and binary gender idealism (Wright 2006; Hakeem 2008).

\section{Gender identity in the social sciences}

Moving away from the clinical literature towards the social sciences, our understanding of gender identity has been furthered by the work of Judith Butler (overviewed in Watson 2005). In Gender Trouble, Butler described gender categories as being fictional products, 'performative' effects of society rather than a property located within an individual (Butler 1990). Butler suggested gender to be an enforced, stylised, cultural performance perpetuated by society. The ritualised repetition of this performance gives the meanings that the particular society affords to gender the illusion of being 'real'. This 'reality' feeds back into the society, perpetuating the cycle of repetition.

\section{Assessment}

At our clinic we believe that it is helpful to both patient and referrer to maintain very few exclusion criteria for the psychotherapy service. We exclude only those with current florid psychosis or significant substance dependence, either of which would compromise the patient's ability to use the therapeutic intervention. Even though such individuals are not offered the group psychotherapy, we may offer them a number of individual consultations to talk with them about their presenting gender confusion. At assessment, the clinical history includes careful detail regarding sex at birth 
BOX 1 Differential diagnosis

- Transvestism Interest in the outer appearance or clothing of the opposite sex

- Fetishistic transvestism Sexual excitation in the clothes of the opposite sex

- Autogynaephilia Sexual excitement from the fantasy of having female bodily parts as a man

- Homosexuality/lesbianism Confusion regarding sense of gender can result from an attraction to members of the same sex felt by the patient to be unacceptable

(biological sex), gender identity and sexual and relationship history. This is essential to discern whether the presenting gender-related symptom is solely located in gender identity or is linked primarily to sexual interests or feelings that cause the patient distress (Box 1).

As Table 1 indicates, almost two-thirds of the patients in the service consider themselves to be pre-operative transsexuals. A quarter of the patients referred are post-operative transsexuals with a persisting sense of dissatisfaction or gender dysphoria after physical sex reassignment. Just over a quarter of patients are referred as having a gender identity disorder but, after assessment, are considered to be transvestites rather than transgender. The ratio of biological males to females referred to the service is approximately six to one.

The clinical history also requires careful attention to the patient's understanding of their sense of gender identity spanning from early childhood to the present, including any periods when the sense of gender was concordant with their biological sex. It is not uncommon for patients initially to attempt to provide what I refer to as 'the official version' of their history, a 'cut and paste' account they have prepared to accord with what they anticipate professionals in gender clinics need to hear in order for them to gain access to physical treatments. If a patient does provide such a generic template of a history, then reassurance on the part of the assessor may encourage the individual to give a more accurate account.

\section{Aims of treatment}

A careful history enables the current gender-related presenting symptom to be understood in context. In addition, a discussion with the patient will identify their particular goals for psychotherapy. The aims of the therapy are psychological rather than physical, and it takes place irrespective of any physical changes the patient may be undergoing or planning to proceed with during the therapy.
The psychotherapy has two explicit goals. The first is to give the patient a greater understanding of the meaning they afford to gender as a construct in relation to themselves and society. The second is to enable the patient to attain a stability, acceptance and satisfaction with their individually tailored gender role, which may or may not correlate with their biological sex, and may or may not fit within a binary framework of gender. Achieving a greater sense of stability and security in their sense of their own gender (irrespective of their biological sex) will help them, regardless of whether they choose surgical alteration of their physical sex.

Once the aims are agreed by both therapist and patient, the model and setting of the proposed course of psychotherapy are explained.

\section{Therapeutic model and delivery}

Following the assessment, the patient is offered a few individual consultations. The number of consultations is tailored to their assessed needs. The main purpose of these sessions is to allow the patient to establish a therapeutic relationship with the therapist before they join a psychotherapy group. The small-group psychotherapy sessions are run weekly and last $75 \mathrm{~min}$. The groups are classed as 'slow open', having a set membership (up to eight patients) whose places are filled as members of the group are discharged. As the service operates within a clinic that is still able to offer time-unlimited psychotherapy, the overall duration of treatment is determined by the patient, informed by their response to, and benefit from, the therapy. Most patients in the service find that their presenting difficulties relating to their sense of gender identity are satisfactorily addressed within the first 2 years of being in a group but choose to remain in the group for longer. Determining an ideal length of treatment in order for the service to be replicated elsewhere will be possible once a tailored evaluation tool for objectively measuring therapeutic response has been created and validated. This will be discussed later.

The group sessions involve a modified group analytic psychotherapy incorporating elements of mentalisation-based therapy (Box 2). Group

B0X 2 Mentalisation

Mentalisation is the ability to recognise and understand feelings, intentional states and mental processes within ourselves, in others and in interpersonal contexts. Mentalisation-based therapy as devised by Bateman \& Fonagy (2004) is a specifically tailored therapy aimed at increasing a person's ability to mentalise. 
analysis (group analytic therapy) was devised by the psychiatrist S. H. Foulkes in the early 1970s and has its roots in psychoanalysis, social theory and systems theory (Foulkes 1964). Mentalisationbased therapy has primarily been developed for use in borderline personality disorder (Bateman 2004). In psychotherapy for gender identity disorders, mentalisation involves working collaboratively with patients towards a greater understanding and clarity of their reported feelings or convictions regarding their gender. Unless the patient has an additional diagnosis of borderline personality disorder, this is usually the only mentalisation deficit to be addressed. However, most patients initially struggle to envisage the meanings afforded to gender by themselves and others. This genderfocused 'non-mentalisation' includes mentalisation of how others may perceive or attribute meanings regarding the patient's gender. The binary nature of the gender conflict, along with the social nature of gender, make group therapy the preferred approach. In individual therapy, therapists can find themselves drawn into a protagonist/ antagonist dynamic with the patient that is very much like the male/female binary dyad.

Analytic therapy groups can be classified as either 'homogeneous' or 'heterogeneous', depending on whether the presentations of patients in the group are similar or different (Foulkes 1975). As all the patients in our gender identity groups have or have had an issue relating to their sense of gender identity, the groups would be considered to be homogeneous. The rationale for running a homogeneous group for people with gender identity disorders was to avoid the risk that the gender conflict would be under- or overattended to in a heterogeneous group. In a homogeneous group, in which the conflict is present in some manifestation in all members, it may more comfortably take a foreground or background position.

As indicated in Table 1, groups may include individuals who wish to pursue sex reassignment surgery, those who have already had surgery and those who feel transgendered but have no intentions to have surgery. At the gender identity clinic, my transgender groups also include patients who are not easily categorised within a pre-operative or post-operative framework. Some show diagnostic fluidity between the categories of transsexual and transvestite. There are also rare cases of autogynaephilia in which the patient wishes to retain a male gender identity but with female genitalia or other female physical parts. Thus, although the group at first sight might appear to be homogeneous it may be considered to be heterogeneous.

\section{Specialist adaptation of therapeutic technique}

There is often much confusion among both patients and professionals regarding the terms 'sex' and 'gender'. It is probably easiest if the biological, phenotypic and chromosomal sex differences of 'male' and 'female' are considered as having an organic basis, in contrast to the role meanings afforded to these sexes in the form of 'masculine' and 'feminine' genders, which are social constructs. Gender is a psychosocial virtual entity without location in a person's body. It solely exists in, and is perpetuated by, the society in which the person lives.

Patients referred with gender identity conditions frequently have a rigid, binary perception of gender. They feel that they do not comfortably fit into this framework and therefore feel persecuted by it. This mismatch usually leads them to question the validity of their own gender, rather than that of society's gender classification framework. In contrast, gender-confident individuals may comfortably play with or subvert societal gender frameworks without experiencing any insecurity in their own sense of gender identity. Such mixing and matching of elements from across the perceived binary divide is seen in the recent phenomena of the 'metro-sexual' male and the female 'lad-ette'.

In psychotherapy for gender identity disorder, the clinician's task is to deconstruct rigid gender frameworks perceived by the patient, so as to open up a range of what is possible for the individual in any given gender role.

\section{Deconstructing a binary gender rigidity}

When a patient uses the terms 'masculine' or 'feminine', their understanding of these words should not be presumed. Rather, it should be explored in the therapy within a mentalisation framework. The aim is not to discourage use of the terms, but instead to encourage a degree of enquiry as to what they mean to those using them (mentalisation), rather than presume a universally understood meaning (non-mentalisation).

Although patients may refer to 'male' or 'female' clothes, attitudes, mannerisms, thinking styles and interests, it is the task of the therapist in this specially adapted therapeutic work to 'degender' the things that patients have 'gendered' in their minds. Long-standing 'gendering' adds to identity confusion when individuals find themselves mismatched with the perceived gendered framework. If aspects of themselves are identified as qualities they had classified as 'male' or 'female', a gender conflict will be reinforced if these are incongruent with their biological sex. 
As regards clothing in particular, the therapist should maintain that, although the patient or the society in which they live assign gender associations to particular items of clothing, these are merely societal constructions. Therefore an interest in any particular garment does not impart a greater or lesser validity to any particular gender identity. Patients should be encouraged to wear to sessions whatever they would normally wear and any gendered associations they may have afforded to their clothes can then be considered within the exploratory arena of the therapeutic group.

Gradually, pre-existing terms and conditions of gender are deconstructed, with the therapeutic aim of reducing the primacy afforded to gender as a construct variable to a level afforded to more benign (in this context) variables such as age, class, race or ethnicity. The rationale behind this is that if patients' pre-existing heightened awareness of gender is lessened to the extent that it no longer occupies an overriding focus, there will be a concomitant reduction in their gender-related dissatisfaction and psychopathology.

\section{Features specific to psychotherapy for gender identity}

In addition to the special adaptation of therapeutic technique required for working with gender identity disorder, I have observed a number of features specific to this field during the running of the specialist service discussed here. These are listed in Box 3 and described below.

\section{Binary rigidity}

A rigid adherence to a binary system of gender rules is core to gender identity disorders (Hakeem 2010a). It is common for patients to give a history of 'not feeling right' early in the evolution of their gender identity disorder. The mismatch between their interests and characteristics and what they perceive society to dictate to be 'appropriate' for their biological sex serves as confirmation to the patient of their transgender or other gender identity disorder. A similar 'not feeling right' is often reported by patients for whom sex reassignment surgery did not provide the psychological solution

BOX 3 Observed features specific to gender identity psychotherapy

- Binary rigidity

- Genital centrality

- Confusion

- Questioning of authenticity they had hoped for and who once again find themselves in a body that they feel is wrong.

During therapy, it is common for patients abruptly to switch to a transgender presentation and just as abruptly switch back in the following session. Similarly common is for a post-operative transgender patient suddenly to return to the gender presentation of their original biological sex, or to oscillate between these binary gender presentations. Such abrupt switching is more often observed at the early stages of therapy, perhaps as the patient acclimatises to the process of gender exploration. It is an example of exploration occurring on the physical level before it moves to the psychological one. Switching between binary gender 'solutions' should be tolerated and its meanings considered with the patient.

In the field of gender identity, binary rigidity is not confined to patients or to gender rules. Both patients and professionals often find themselves drawn into occupying - or misperceived as occupying - binary stances on a number of politically charged topics in relation to gender identity. Most commonly, professionals are perceived, accurately or inaccurately, as adopting either 'advocate' or 'protagonist'positions in relation to sex reassignment surgery. Not only individuals but entire institutions come to be seen, sometimes completely unjustly, as rigidly adopting such binary stances. For some years, the specialist gender identity psychotherapy service at the clinic was perceived by many patients external to it to be 'transphobic' for offering psychotherapy rather physical (hormonal/surgical) treatment. This is at odds with the experience of the patients within the service, many of whom receive both psychotherapeutic and physical interventions concurrently from respective clinics and benefit from both. I would advise clinicians entering this field to be aware of - and avoid being drawn into - such binary positions with regard to transgender politics. I believe that maintaining an open mind and a position of neutrality, in combination with scientific curiosity and enquiry regarding the presenting problems, is the clinician's duty to this patient population.

\section{Genital centrality}

For many people seeking professional help for gender identity disorders, the presenting problem is that their genitalia 'are wrong', rather than a sense of dissatisfaction, confusion or particular emotional response in relation to their genitalia. Many patients are clear in their minds that the primary problem is located in the sex of their body and that the psychological problems they report are secondary to this, rather than vice versa. 
Such genital centrality is not restricted to the patients. The gender identity service that I run used to operate two separate therapy groups, one for pre-operative and one for post-operative transsexuals. The pre-operative group was characterised by hope and optimism, at times extending to a gender euphoria, whereas the postoperative group was characterised by despair, hopelessness and regret, mainly because the group members were individuals who wished they had not had surgery. Our arbitrary classifying of patients on the basis of whether or not they had had sex reassignment surgery mirrored the genital centrality observed in many of our patients.

Psychotherapists working outside the service frequently ask whether people with gender identity disorders who are focused on physical solutions should be offered psychotherapy. Some even suggest that 'nothing can be done' for postoperative transsexuals not helped by genital surgery (Hakeem 2010b). There is no evidence to support such a speculative correlation of the presence of intact genitalia with the ability to benefit from psychotherapeutic interventions.

Again, the genital focus of such unfounded concerns appears to mirror the focus of many patients with gender identity disorders.

\section{Confusion}

Many individuals with gender identity disorders have fixed cross-gendered identities at the time of referral, but develop an increasing degree of fluidity and confusion in their understanding of their own and others' genders during therapeutic enquiry and exploration. Such confusion is not always confined to the patients. Therapists must be prepared for patients who present themselves in different gender roles, sometimes switching abruptly from one session to the next. It can be a challenge to face a familiar, yet unfamiliar patient. The confusion can also extend to other parts of the institution: administrative staff have to contend with changing gender pronouns, and management may be unsure which toilets patients should be allowed to use in the building.

\section{Questioning of authenticity}

A common feature in the clinical history of people with gender identity disorders is their concern regarding the authenticity of their gender role. Patients presenting with a sense of confusion or unhappiness with their biological sex frequently report not living up to what they believed was expected of them in the corresponding gender role. In their early years, this questioning of the authenticity of their gender role may initially have been by other people, but they later take it on themselves. Unsurprisingly, a rigid adherence to perceived binary gender roles with which the individual feels mismatched is often a precursor. Subsequently, they question whether they could or should be 'authentically' considered a member of the sex corresponding to the gender role or, indeed, whether a greater sense of legitimacy could be achieved by changing their gender role.

Physical sex reassignment provides a lasting solution to a mismatched gender identity for many patients with transgender problems. However, it is certainly not a universal solution and this is the reason for the careful assessment process carried out by gender identity clinics (Eden 2012). As I have already mentioned, about a quarter of the patients I see for psychotherapy have undergone sex reassignment surgery and have later regretted it. These individuals also report a sense of inauthenticity with regard to their sense of gender identity and once again report 'not feeling right' with the body they now have. Some give a history of unrealistic hopes of becoming the opposite sex, but of never being accepted by others as an 'authentic' woman (or man) after surgery. The challenge to their authenticity in their new gender may not be external. Many successfully 'pass' in society: they look, and on the surface behave, like the man or woman they have chosen to be. However, they do not 'read' themselves as belonging to that gender. They report that the challenge comes from within themselves, as they have come to question the certainty they once had regarding their transgender identity.

\section{Parallel processes}

In discussing the four features specific to gender identity disorder in the previous section, I repeatedly noted that a particular feature was evident not only in the patient, but could sometimes also be seen in the therapist, the organisation and people outside of the clinic. One example that I give is that of genital centrality, where patients, specialist clinics and non-specialist psychotherapists evaluate or classify individuals in terms of their genitalia. This phenomenon reflects what in psychotherapy are called 'parallel processes': features of either the cognitive style or the pattern of interpersonal relationships of patients in therapy that are also observed (in parallel) in the wider context in which the therapy is delivered (Hakeem 2010b).

\section{The next step: the need for a validated evaluation tool}

As mentioned earlier, the national psychotherapy service for people with gender identity disorder 
was originally established as a feasibility pilot. I had no idea whether patients would want such a service or find it useful. The response, however, has been striking. Patients who have gone through the therapeutic programme have reported that it has been useful in its specific focus on understanding themselves in relation to their sense of gender identity.

Obviously, such patient-reported outcomes are anecdotal evidence only. The next stage in the development of this specialist intervention will be to support the patient feedback and our observations by an evidence-based qualitative and quantitative outcome study. The initial task faced is to create a valid and reliable evaluation tool to measure satisfaction with and stability of gender identity irrespective of whether this correlates with biological sex. At present there are no such tools to measure gender stability or sustained gender identity. As outlined here, adherence to a perceived rigid binary gender framework is unhelpful and, at times, iatrogenic for people with gender identity disorder. Consequently, any evaluation tool would have to avoid restricting gender identities to a binary repertoire. It would need to allow for fluid and creatively tailored gender identities such as genderqueer and other non-binary gender options.

\section{Conducting an outcome study}

The evaluation measure should be administered at first assessment, at intervals during therapy, at the end of the therapy programme and again at follow-up. Using an assessment tool at intervals during treatment would enable an optimum length of psychotherapy to be determined. Patients should be followed up after the intervention has ended to ascertain whether their gender stability is sustained.

Referrers and any other professionals involved in the care of the patients I see at the gender identity clinic are sent update reports three times a year and patients are eventually discharged back to their general practitioner. They are referred to secondary psychiatric services only if they have pre-existing psychiatric illnesses requiring this. Patients are able to request a follow-up appointment or re-referral to the service, but no one has yet done so.

\section{Conclusions}

People with gender identities that are less sustained or do not fit into a binary male/female gender framework, those who do not wish to pursue physical treatments and those for whom sex reassignment has been unsuccessful in addressing their gender dysphoria may all be suitable for a specially adapted group psychotherapy (Box 4).
BOX 4 Key learning points

- Not all gender identity disorders are typical transsexualism/transgender

- Hormonal and surgical sex reassignment are useful for some but not all gender identity disorders

- For individuals with atypical gender identity disorders, a specially adapted group psychotherapy focusing on gender identity may be useful

- Features specific to the adapted model of psychotherapy include a collaborative enquiry into perceived gender constructs

- Particular features observed as specific to this work include the themes of binary rigidity, genital centrality, rejection, confusion and the questioning of authenticity of gender roles

- Professionals need to be wary of being drawn into polarised stances in relation to transgender politics and instead retain therapeutic neutrality and an open mind

Psychotherapy and physical treatments are not mutually exclusive and many patients wish to pursue both options concurrently. The psychotherapy described in this article has clearly defined, explicit aims that are discussed and agreed with the patient at the outset and worked towards collaboratively throughout the treatment. The aim for patients is to acquire a greater sense of satisfaction and stability in their chosen gender, irrespective of whether this correlates with their biological sex. Through a careful exploration in group therapy of the meanings that group members have afforded to gender in themselves and others, each patient is encouraged to determine an individually tailored gender identity experienced as authentic to themselves. Patients are guided against attempts to assimilate into a perceived rigid binary gender framework, as this can result in a stereotyped caricature and may be iatrogenic.

Therapists working in gender identity disorder need to adopt an open and accepting attitude: a formulaic approach can interfere with their understanding of patients' difficulties. Both therapists and patients need to differentiate between the concepts of 'sex' and 'gender', the former having an organic basis and the latter being a psychosocial construct. Therapists must be open and accepting of patients, who may not conform to a binary heterosexist framework of gender identity and whose identities may be more fluid and indeterminate.

Clinicians should expect to encounter certain recurrent themes specific to working with gender identity disorder. These include binary rigidity, not only in the patient's relationship to gender, but
MCQ answers

$1 \mathrm{c} \quad 2$ e 3 c 4 e 5 e 
also in how professionals may be drawn into (or perceived to be drawn into) polarised positions in relation to transgender surgery and gender politics. Genital centrality is manifest both in the patients' relationships with their bodies and in how non-specialist professionals may categorise patients and their suitability for therapy. Patients referred for psychotherapy often report concerns relating to the authenticity of their gender role. Sometimes, these concerns have remained after physical sex reassignment. Unfortunately, services often deny patients with gender identity disorders the opportunity for psychotherapy, a rejection that is similar to the rejection the patients can show for aspects of themselves, their bodies or gender.

At present, the psychotherapy described here is available in only one clinic in the UK, and many patients have to travel far to access it. The anecdotal evidence supporting this type of therapy deserves to be subjected to empirical study: we believe that this would justify the setting up of similar services elsewhere in the country.

\section{References}

Althof SE, Keller AC (1980) Group therapy with gender identity patients. International Journal of Group Psychotherapy 30: 481-9.

Bateman A, Fonagy P (2004) Psychotherapy for Borderline Personality Disorder: Mentalization-Based Treatment. Oxford University Press.

Blanchard R (2005) Early history of the concept of autogynephilia. Archives of Sexual Behavior 34: 439-446.

Butler J (1990) Gender Trouble: Feminism and the Subversion of Identity. Routledge.

Eden K, Wylie K, Watson E (2012) Gender dysphoria: recognition and assessment. Advances in Psychiatric Treatment 18: 2-11.

Foulkes SH (1964) Therapeutic Group Analysis. George Allen and Unwin.
Foulkes SH (1975) Group Analytic Psychotherapy: Method and Principles. Reprinted (1986) by Karnac Classics: 99-156.

Green R, Fuller M (1973) Group therapy with feminine boys and their parents. International Journal of Group Psychotherapy 23: 54-68.

Greenson RR (1968) Dis-identifying from mother. International Journal of Psychoanalysis 49: 370-4.

Hakeem A (2007) Trans-sexuality: a case of the emperor's new clothes? In Lectures on Violence, Perversion and Delinquency: The Portman Papers (eds S Ruszczynsky, D Morgan): 179-92. Karnac.

Hakeem A (2008) Changing sex or changing minds: specialist psychotherapy and transsexuality. Group Analysis 41: 182-96.

Hakeem A (2010a) Deconstructing gender in trans-gender identities. Group Analysis 43: 141-54.

Hakeem A (2010b) 'Parallel processes': observed in the patient, therapy and organization. Group Analysis 43: 523-38.

Limentani A (1989) Between Freud and Klein: The Psychoanalytic Quest for Knowledge and Truth. Karnac.

Lothstein LM (1977a) Psychotherapy with patients with gender dysphoria syndromes. Bulletin of the Menninger Clinic 41: 563-82.

Lothstein LM (1977b) Countertransference reactions to gender dysphoric patients: implications for psychotherapy. Psychotherapy: Theory, Research and Practice 14: 21-31.

Lothstein LM (1979) Group therapy with gender-dysphoric patients. American Journal of Psychotherapy 33: 67-81

Lothstein LM, Levine SB (1981) Expressive psychotherapy with gender dysphoric patients. Archives of General Psychiatry 38: 924-9.

Stoller R (1968) Sex and Gender: The Development of Masculinity and Femininity. Karnac.

Stoller R (1975) Sex and Gender. Vol. II: The Transsexual Experiment. Hogarth Press and Institute of Psychoanalysis.

Watson K (2005) Queer theory. Group Analysis 38: 67-81.

World Health Organization (1992) The ICD-10 Classification of Mental and Behavioural Disorders: Clinical Descriptions and Diagnostic Guidelines. WHO

Wright J (2006) The remains of the day: transsexuality, psychodynamics and the modern NHS. Psychoanalytic Psychotherapy 20: 140-56.

Wylie K, Eden K, Watson E (2012) Gender dysphoria: treatment and outcomes. Advances in Psychiatric Treatment 18: 12-16.

\section{MCOs}

Select the single best option for each question stem

1 Individuals with a changeable and uncertain sense of gender identity disorder should be offered:

a cross-sex hormones

b sex reassignment surgery

c psychotherapy

d behavioural conditioning

e antipsychotics.

\section{The adaptation of psychotherapy} described here incorporates elements of:

a psychoanalytic psychotherapy

b mentalisation-based therapy

c group therapy

d cognitive-behavioural therapy

e both mentalisation-based and group therapy.
3 Psychotherapy for gender identity disorder should be offered:

a only to pre-operative transsexuals

b only when sex reassignment has not helped the patient

c to any patient who feels they could benefit from it

d as an obligatory component of physical sex reassignment

$\mathrm{e}$ if there is psychiatric comorbidity with other mood disorders.

4 The aim of specialist psychotherapy for gender identity disorder is to:

a assist patients progress through sex reassignment

b discourage patients from having sex reassignment

c enable patients to socialise $\mathrm{d}$ teach and reinforce gender-appropriate behaviour

e increase the stability and satisfaction of the patient's sense of their gender, regardless of their biological sex and societal gender frameworks.

5 Common themes observed in the work of gender identity psychotherapy are:

a binary gender rigidity

b genital centrality

c questioning of authenticity

d confusion

e all of the above. 